\title{
RADIO IMAGES OF WOLF-RAYET 147
}

\author{
R.J. DAVIS* \\ NRAO, PO Box O, Socorro, NM 87801, U.S.A. \\ M.F. BODE \\ Astrophysics Group, Liverpool John Moores University, Liverpool L3 3AF, U.K. \\ K.A. VAN DER HUCHT \\ SRON Utrecht, Sorbonnelaan 2, 3584 CA Utrecht, The Netherlands \\ and \\ P.M. WILLIAMS \\ The Observatories, Blackford Hill, Edinburgh, EH9 $3 H J$, U.K.
}

\begin{abstract}
We present preliminary MERLIN radio maps at 1.7 and $5 \mathrm{GHz}$ of the WolfRayet star WR147 (AS431). These provide the highest spatial resolution images of this object at these frequencies to date. Our results confirm that WR147 comprises two primary radio sources lying on a $\mathrm{N}-\mathrm{S}$ axis. Combining results at 1.7 and $5 \mathrm{GHz}$ gives a spectrum for the southerly source consistent with optically thick thermal emission. The northerly source appears to be non-thermal. In addition, extended emission is observed at both frequencies which links the two sources. The northerly source is elongated in a direction roughly perpendicular to the axis of this extended emission, and the southerly source appears double on the $5 \mathrm{GHz}$ map.
\end{abstract}

\section{Introduction}

WR147 is well known to be a bright radio source. Moran et al. (1989), using what is now a partial array of the MERLIN instrument, presented observations which revealed that at $5 \mathrm{GHz}$ the source is double, with a separation between the two components of approximately 0 ". 6 . They conjectured that the southerly source (which is coincident with the stellar position) is thermal, while the northerly component is non-thermal. Subsequently, Churchwell et al. (1992) published results of VLA observations at four frequencies, and their conclusions were broadly in line with those of Moran et al. . However, they also provided a more reliable distance estimate of $630 \mathrm{pc}$, rather than $1.9 \mathrm{kpc}$ as previously assumed. The preliminary observations presented here are part of a larger programme of full MERLIN observations of WR stars.

\section{Observations}

Observations were made at $5 \mathrm{GHz}$ on 1992 June 24 and at $1.658 \mathrm{GHz}$ on 1993 April 11. Twelve hour tracks were used in each case. Since the expected radio flux density from this star is low, a phase-referencing technique (Peckham

* On leave from NRAL, University of Manchester, Jodrell Bank, Macclesfield, U.K. 


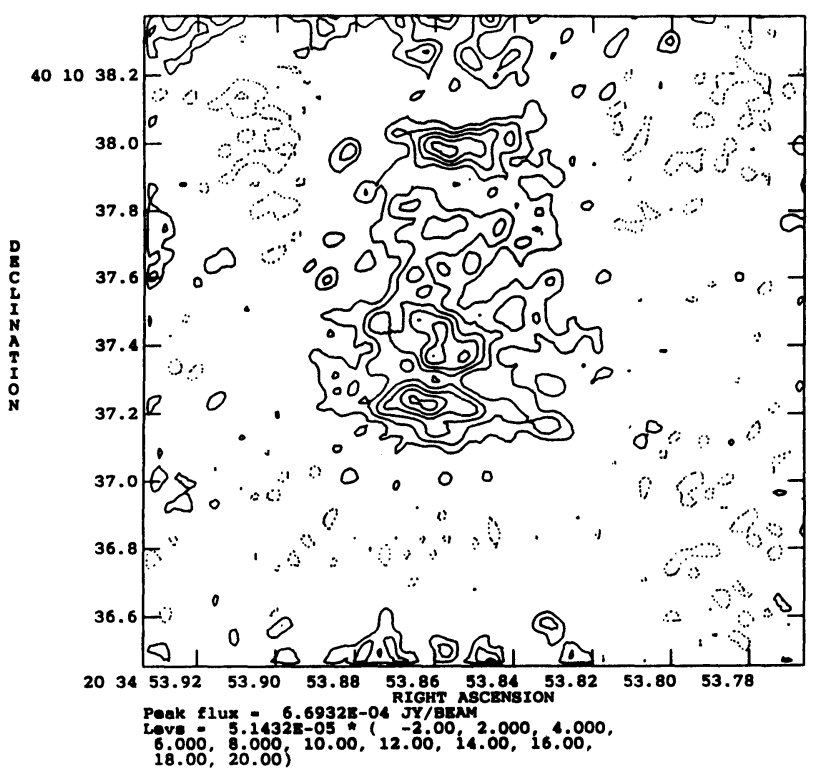

Fig. 1. $5 \mathrm{GHz}$ MERLIN map of WR147 obtained on 1992 June 24, processed using a maximum entropy technique.

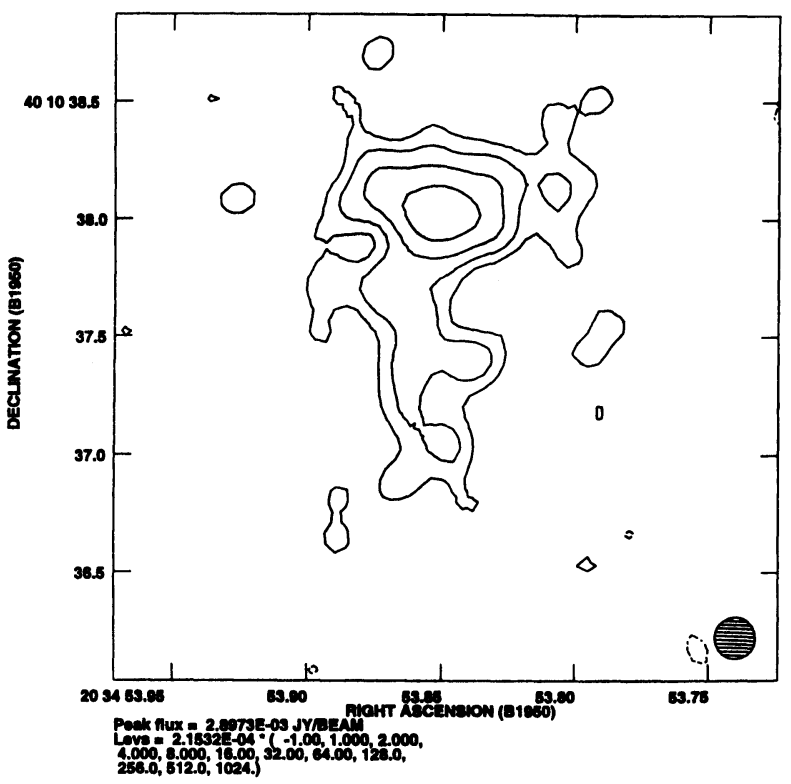

Fig. 2. $1.7 \mathrm{GHz}$ MERLIN map of WR147 obtained on 1993 April 11 
1973) was used in which twelve minute observations of WR147 were interspersed with three minute observations of a phase-reference source. The data were edited, vector-averaged and calibrated using routines in the OLAF (Off Line Analysis Format - an astronomical image processing package that is designed specifically to process MERLIN or VLBI data) software package and the visibility data were transformed to an intensity map using the Astronomical Image Processing System (AIPS). Initially the phase-reference source was mapped by the standard self-calibration technique (Schwab 1980). The derived telescope gain solutions were then applied to WR147 and the resulting data were further processed by Fourier and CLEAN techniques (Hogbom 1974). Using these techniques, the flux density and position of the source are accurately related to that of the phase reference. The flux density at 5 $\mathrm{GHz}$ of the reference source $2005+403$ was determined to be $4.4 \mathrm{Jy}$ from a comparison observation of the calibration source 3C286, whose flux density was assumed to be $7.4 \mathrm{Jy}$.

\section{Results}

The resulting map at $5 \mathrm{GHz}$, with a beam of $0^{\prime \prime} .05 \times 0 .{ }^{\prime \prime} 05$ is shown in Fig.1. As can be seen, the source is clearly resolved into two primary components, separated by $\approx 0^{\prime \prime} .6$. The new, high resolution map suggests that the southern source itself is double. In addition, a complex extended structure appears to join the two primary sources.

Fig. 2 shows preliminary observations of the star at $1.7 \mathrm{GHz}$ obtained with the MERLIN system in phase-reference mode. These results indicate that the northerly source is elongated normal to the axis joining the two sources. This may be the first spatially resolved radio image of a non-thermal component in a WR star. The emission presumably arises from some form of shock-interaction. All the other emission on the maps appears to be thermal. Indeed, Moran et al. showed that the optical stellar source is more reasonably associated with the southerly complex. However, further astrometry is required to add confidence to this result. All the data at both frequencies have been recalibrated with the new MERLIN calibration package and much improved maps will be provided in the near future.

\section{References}

Churchwell, E.B., Bieging, J.H., van der Hucht, K.A., Williams, P.M., Spoelstra, T.A.Th., Abbott, D.C. 1992, ApJ 393, 329

Hogbom, J.A. 1974, A\&A Suppl. 15, 417

Moran, J.P., Davis, R.J., Bode, M.F., Taylor, A.R., Spencer, R.E., Argue, A.N., Irwin, M.J., Shanklin, J.D. 1989, Nature 340, 449

Peckham, R.J. 1973, MNRAS 165, 25

Schwab, F. 1980, Proc. Soc. Photo-Optical Instr. Engin. 231, 18 